\title{
Magnetism and cluster glass dynamics in geometrically frustrated $\mathrm{LuFe}_{2} \mathrm{O}_{4}$
}

M. H. Phan, N. A. Frey, H. Srikanth, M. Angst, B. C. Sales, and D. Mandrus

Citation: Journal of Applied Physics 105, 07 E308 (2009);

View online: https://doi.org/10.1063/1.3074507

View Table of Contents: http://aip.scitation.org/toc/jap/105/7

Published by the American Institute of Physics

\section{Articles you may be interested in}

Charge ordering, ferroelectric, and magnetic domains in $\mathrm{LuFe}_{2} \mathrm{O}_{4}$ observed by scanning probe microscopy Applied Physics Letters 106, 152902 (2015); 10.1063/1.4918358

Strong spin-lattice coupling in $\mathrm{CrSiTe}_{3}$

APL Materials 3, 041515 (2015); 10.1063/1.4914134

Ferroic investigations in $\mathrm{LuFe}_{2} \mathrm{O}_{4}$ multiferroic ceramics

Journal of Applied Physics 110, 034108 (2011); 10.1063/1.3622147

Oxygen stoichiometry and magnetic properties of $\mathrm{LuFe}_{2} \mathrm{O}_{4+\delta}$

Journal of Applied Physics 113, 063909 (2013); 10.1063/1.4792036

Atomic-resolution chemical imaging of oxygen local bonding environments by electron energy loss spectroscopy Applied Physics Letters 101, 042907 (2012); 10.1063/1.4737208

Room temperature giant dielectric tunability effect in bulk $\mathrm{LuFe}_{2} \mathrm{O}_{4}$ Applied Physics Letters 92, 182903 (2008); 10.1063/1.2920775

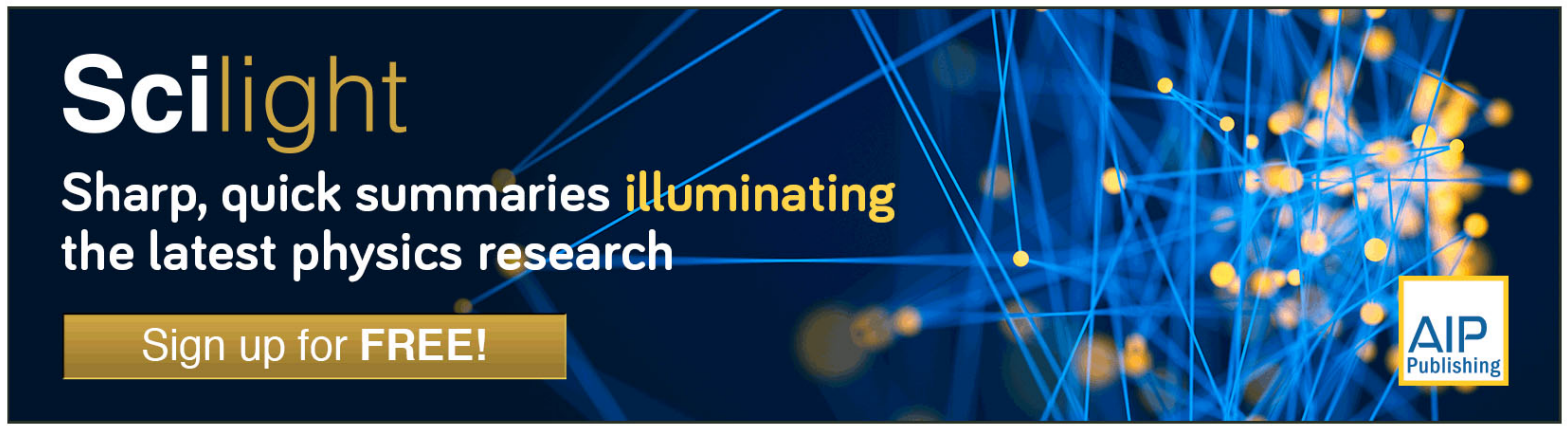




\title{
Magnetism and cluster glass dynamics in geometrically frustrated $\mathrm{LuFe}_{\mathbf{2}} \mathrm{O}_{\mathbf{4}}$
}

\author{
M. H. Phan, ${ }^{1}$ N. A. Frey, ${ }^{1}$ H. Srikanth, ${ }^{1, a)}$ M. Angst ${ }^{2, b)}$ B. C. Sales,${ }^{2}$ and D. Mandrus ${ }^{2}$ \\ ${ }^{1}$ Department of Physics, University of South Florida, Tampa, Florida 33620, USA \\ ${ }^{2}$ Materials Science and Technology Division, Oak Ridge National Laboratory, Oak Ridge, Tennessee 37831, \\ USA
}

(Presented 14 November 2008; received 12 September 2008; accepted 9 December 2008; published online 19 February 2009)

\begin{abstract}
We report on the magnetic properties of high quality $\mathrm{LuFe}_{2} \mathrm{O}_{4}$ single crystals grown by the floating zone method. dc and ac susceptibility measurements and analysis reveal a ferrimagnetic transition at $\sim 240 \mathrm{~K}$ followed by a re-entrant cluster glass transition below $225 \mathrm{~K}$, with an additional magnetic transition around $170 \mathrm{~K}$. Strong frequency dependence of the real $\left(\chi^{\prime}\right)$ and imaginary $\left(\chi^{\prime \prime}\right)$ parts of the ac susceptibility observed at both these temperatures indicate glassy behavior and we quantitatively fit the data to a cluster glass model, $\tau=\tau_{\mathrm{o}}\left(T_{f} / T_{g}-1\right)^{-z v}$. Our studies show that these multiple transitions are consistent with the picture of ferrimagnetic clusters in the iron oxide planes with triangular lattice configuration favoring spin frustration and glass dynamics.
\end{abstract}

(C) 2009 American Institute of Physics. [DOI: 10.1063/1.3074507]

Charge frustrated compounds $R \mathrm{Fe}_{2} \mathrm{O}_{4}(R=\mathrm{Y}, \mathrm{Er}, \mathrm{Yb}$, $\mathrm{Tm}$, and $\mathrm{Lu}$ ) have received growing attention owing to their extraordinarily interesting physical properties. ${ }^{1}$ Among them, $\mathrm{LuFe}_{2} \mathrm{O}_{4}$ is of topical interest as it has been shown that ferroelectricity in this material arises from electron correlation effects (i.e., iron $\mathrm{Fe}^{2+}-\mathrm{Fe}^{3+}$ valence ordering) rather than conventional covalency. ${ }^{2-4}$ Giant magnetocapacitance effects have been observed at room temperature rendering it multiferroic and also pointing toward an intricate coupling between the magnetic, electronic, and structural degrees of freedom. ${ }^{5,6}$ However, a good understanding of the magnetic phase diagram has remained elusive primarily due to the complexity of the system as well as the sensitivity to sample quality. ${ }^{7-10}$ While Iida et $a .^{8}$ showed the absence of a longrange three dimensional (3D) magnetic order down to $4.2 \mathrm{~K}$ in $\mathrm{LuFe}_{2} \mathrm{O}_{4}$ thus suggesting that this system at low temperatures consists of ferromagnetic clusters of various sizes, recent neutron diffraction studies have revealed the existence of a magnetic 3D correlation (e.g., a long-range magnetic order) even below $175 \mathrm{~K}$, where a magnetic transition which is associated with a structural transition at the same temperature takes place. ${ }^{9}$ In contrast to these studies, Wang et al. ${ }^{10}$ argued that $\mathrm{LuFe}_{2} \mathrm{O}_{4}$ undergoes a paramagnetic (PM) to ferrimagnetic $(\mathrm{FM})$ transition around $\sim 236 \mathrm{~K}$ followed by a re-entrant spin glass (SG) transition below $\sim 228 \mathrm{~K}$.

To elucidate these features further, we have studied the magnetic properties of high quality $\mathrm{LuFe}_{2} \mathrm{O}_{4}$ single crystals. Our dc and ac magnetic measurements reveal the presence of multiple transitions in $\mathrm{LuFe}_{2} \mathrm{O}_{4}$ and these transitions are consistent overall with the picture of ferrimagnetic clusters in the iron oxide planes with the triangular lattice configuration favoring spin frustration and glass dynamics.

\footnotetext{
${ }^{\text {a) }}$ Author to whom correspondence should be addressed. Electronic mail: sharihar@cas.usf.edu.

${ }^{b)}$ Present address: Institut für Festkörperforschung, Forschungszentrum Jülich GmbH, D-52425 Jülich, Germany.
}

$\mathrm{LuFe}_{2} \mathrm{O}_{4}$ single crystals were grown by an image furnace floating zone technique, using an oxygen partial pressure tuned by a $\mathrm{CO} / \mathrm{CO}_{2}$ mixture as described in Refs. 4 and 9; x-ray diffraction confirmed the absence of any impurity phases. ${ }^{4,9}$ The temperature dependences of the zero-fieldcooled (ZFC) and field-cooled (FC) magnetization were measured using a physical property measurement system (PPMS) from Quantum Design in the temperature range of 5-300 K at applied fields up to $70 \mathrm{kOe}$. The PPMS was also used for ac susceptibility measurements, and both the real $\left[\chi^{\prime}(T)\right]$ and imaginary $\left[\chi^{\prime \prime}(T)\right]$ components of the susceptibility were simultaneously measured while warming up from $5 \mathrm{~K}$. All the magnetic measurements reported in this paper were performed with the field along the $c$ axis of the $\mathrm{LuFe}_{2} \mathrm{O}_{4}$ crystal.

Figure 1 shows the ZFC and FC magnetization curves taken at 100 Oe applied field. Multiple magnetic transitions can be clearly discerned in the data. The onset from paramagnetic to ferrimagnetic state occurs at around $240 \mathrm{~K}$ [also seen clearly in plots of inverse susceptibility $(H / M)$ vs $T$, not shown in this paper] followed by a sharp peak in the ZFC curve at $\sim 225 \mathrm{~K}$ and a broader feature around $170 \mathrm{~K}$. Distinct changes in slopes at these temperatures are also ob-

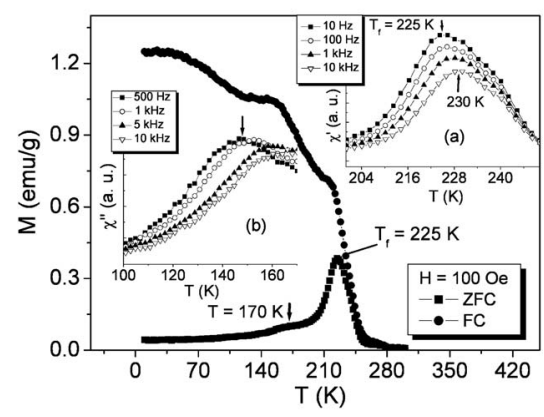

FIG. 1. Temperature dependence of FC and ZFC magnetization taken at $H=100$ Oe. The insets (a) and (b) show the $\chi^{\prime}(T)$ and $\chi^{\prime \prime}(T)$ curves for representative frequencies $(f=10 \mathrm{~Hz}-10 \mathrm{kHz})$ in the two temperature ranges of around 225 and $175 \mathrm{~K}$, respectively. 
served in the FC curve. While the peak in the ZFC magnetization in the past has been interpreted as due to an antiferromagnetic transition, ${ }^{7,8}$ we will show below that this feature as well as the broader peak at $170 \mathrm{~K}$ is associated with the $\mathrm{LuFe}_{2} \mathrm{O}_{4}$ undergoing magnetic glass transitions at these temperatures.

The glassy behavior in $\mathrm{LuFe}_{2} \mathrm{O}_{4}$ is revealed in ac susceptibility measurements that are widely used to study glass transitions in a variety of materials. ${ }^{10-13}$ The temperature dependences of the real $\left[\chi^{\prime}(T)\right]$ and imaginary $\left[\chi^{\prime \prime}(T)\right]$ components of the susceptibility of $\mathrm{LuFe}_{2} \mathrm{O}_{4}$ were measured at different fixed frequencies ranging from $10 \mathrm{~Hz}$ to $10 \mathrm{kHz}$. In these measurements, the amplitude of the ac field was kept constant at $h_{\mathrm{ac}}=10$ Oe. We have found that at $\sim 240 \mathrm{~K}$, both $\chi^{\prime}(T)$ and $\chi^{\prime \prime}(T)$ rapidly decrease to zero and are independent of frequency, coinciding with the occurrence of the PM-FM phase transition observed in dc magnetization. However, at $T<240 \mathrm{~K}$ there exist two peaks at $\sim 225$ and $\sim 170 \mathrm{~K}$ in $\chi^{\prime}(T)$ and $\chi^{\prime \prime}(T)$ curves and these peaks are strongly frequency dependent. While the peak at $\sim 225 \mathrm{~K}$ is quite sharp, the one at $\sim 170 \mathrm{~K}$ is somewhat broad and the frequency dispersion is much clearer in $\chi^{\prime \prime}(T)$ curves than in $\chi^{\prime}(T)$. The ac susceptibility peaks and their frequency dependence are plotted in the insets (a) and (b) of Fig. 1. It is worth noting that these peaks in $\chi^{\prime}(T)$ and $\chi^{\prime \prime}(T)$ shift to higher temperature and the height of the peak decreases (or increases) as the measurement frequency is increased [see, for example, inset (a) of Fig. 1]. This behavior is characteristic of conventional spin glass systems. ${ }^{11}$ It has been shown that, for a spin glass system, as temperature is decreased below the glass transition, the relaxation time slows down leading to a divergence of the maximum relaxation time at $T_{g}$, where the system enters the spin glass state. ${ }^{11,14}$ The frequency-dependent maximum in $\chi^{\prime}(T)$ denotes the freezing temperature $T_{f}$ where the maximum relaxation time $\tau_{\max }$ of the system is equal to the characteristic time $t=1 / \omega$ set by the frequency of the ac susceptibility measurement. As a result, $T_{f}$ is a function of driving frequency $\omega$. If $T_{f}$ is tuned by varying measurement frequency in a wide frequency range, we are able to determine the maximum relaxation time of the system $\tau_{\max }$ as the spin glass phase is approached by fitting the data to a model representing the conventional critical slowing down given by the expression ${ }^{14}$

$$
\frac{\tau_{\max }}{\tau_{0}}=\left(\frac{T_{f}-T_{g}}{T_{g}}\right)^{-z v},
$$

where $T_{g}$ is the spin glass transition temperature, $z$ is the dynamical exponent, $v$ is the usual critical exponent for the correlation length, and $\tau_{0}$ is the microscopic flipping time of the fluctuating spins. The scaling of the ac susceptibility is plotted in Fig. 2 for the case of the peak at $\sim 225 \mathrm{~K}$, and the best fit to Eq. (1) yields $T_{g} \approx 224.6 \mathrm{~K}, z v \approx 2.85$, and $\tau_{0}$ $\approx 9.18 \times 10^{-8}$ s. A similar procedure using the $\chi^{\prime \prime}(T)$ data was also employed for the case of the peak at $\sim 170 \mathrm{~K}$, yielding $T_{g} \approx 137.5 \mathrm{~K}, z v \approx 3.12$, and $\tau_{0} \approx 9.8 \times 10^{-6} \mathrm{~s}$. In both these cases, the obtained values of $\tau_{0}$ are much larger than typical values for a conventional spin glass system $\left(\tau_{0}\right.$ $\left.\sim 10^{-13} \mathrm{~s}\right),{ }^{11}$ but in good agreement to values found in cluster glass $(\mathrm{CG})$ systems $\left(\tau_{0} \sim 10^{-7}-10^{-9} \mathrm{~s}\right) .^{12,13}$ This quanti-

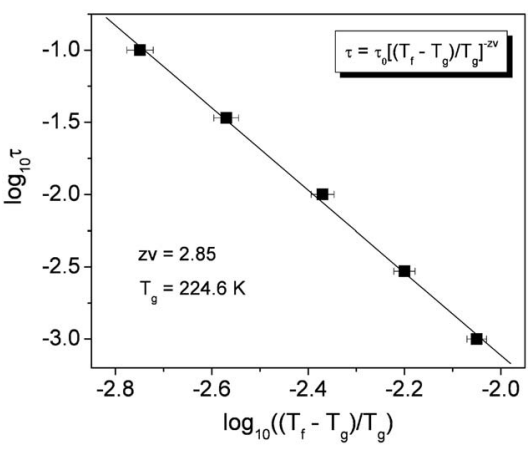

FIG. 2. The best fit of $T_{f}(\omega)$ data extracted from ac susceptibility measurements $\left[\chi^{\prime}(T)\right]$ to the glass model (1) for the case of the peak at $\sim 225 \mathrm{~K}$.

tative analysis indicates that the magnetic phase of the $\mathrm{LuFe}_{2} \mathrm{O}_{4}$ system can be viewed as arising due to an assembly of clusters whose sizes and distribution vary with temperature. The difference in $\tau_{0}$ for the two cases at $\sim 225 \mathrm{~K}\left(\tau_{0}\right.$ $\left.\approx 9.18 \times 10^{-8} \mathrm{~s}\right)$ and $\sim 170 \mathrm{~K}\left(\tau_{0} \approx 9.8 \times 10^{-6} \mathrm{~s}\right)$ clearly suggests a considerable difference in the size and distribution of clusters at these temperatures with the tendency of the average cluster size to increase as the temperature is lowered resulting in much larger relaxation times. Since the magnetic transition at $\sim 175 \mathrm{~K}$ is likely associated with a structural transition which occurs at the same temperature, ${ }^{9}$ it would be logical to infer that the structural transition may lead to a variation in the size and distribution of clusters and hence the spin dynamics in the sample. ${ }^{15}$ However, further study of this structural transition needs to be done to further elucidate any relationship between it and the relaxation dynamics observed in the ac magnetic response. The important fact that clearly emerges for the first time from our study is that the $\mathrm{LuFe}_{2} \mathrm{O}_{4}$ system undergoes a phase transition from a paramagnetic phase to a ferrimagnetic phase at $\sim 240 \mathrm{~K}$ followed by a re-entrant cluster glass transition below $225 \mathrm{~K}$ with an additional magnetic transition that exhibits cluster glass characteristics, also being present around $170 \mathrm{~K}$. We note again that while the peak in ZFC magnetization at $225 \mathrm{~K}$ was interpreted in the past as due to an antiferromagnetic transition, ${ }^{7,8}$ our data and experiments from another group ${ }^{10}$ have now clarified this to be a result of 3D ferrimagnetic ordering followed by a re-entrant glass transition. We however would like to make the distinction that unlike the case reported by Wang et al., ${ }^{10}$ our results clearly show that $\mathrm{LuFe}_{2} \mathrm{O}_{4}$ belongs to a class of $\mathrm{CG}$ materials and not conventional $\mathrm{SG}$ phase. The difference in $\tau_{0}$ between our case $\left(\tau_{0} \approx 10^{-8} \mathrm{~s}\right)$ and their case $^{10}\left(\tau_{0} \approx 10^{-13} \mathrm{~s}\right)$ suggests that the average size and distribution of clusters may vary even in carefully grown single crystals under different conditions since the magnetic properties of $\mathrm{LuFe}_{2} \mathrm{O}_{4}$ depend strongly on oxygen stoichiometry and sample quality. ${ }^{7-10}$ This may also be reconciled with the fact that the magnetic transition around $175 \mathrm{~K}$ is only reported in single crystals synthesized at the ORNL (the source for our studies as well as the synchrotron and neutron scattering experiments of Refs. 4 and 9) but absent in single crystals studied by Wang et al. ${ }^{10}$

Finally, to get a slightly different perspective of the magnetic phase transitions in $\mathrm{LuFe}_{2} \mathrm{O}_{4}$, the measured data of the 


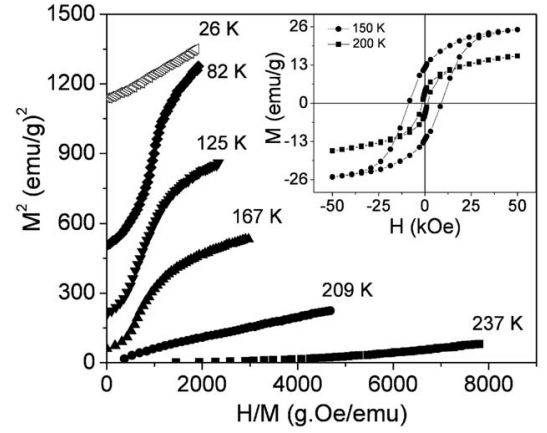

FIG. 3. Arrott plots of magnetization curves for representative temperatures of $26,82,125,167,209$, and $237 \mathrm{~K}$. The inset shows magnetic loops at selected temperatures of 200 and $150 \mathrm{~K}$, indicating a strong increase in $H_{c}$ at temperatures below $175 \mathrm{~K}$

$M-H$ isotherms were converted into $H / M$ vs $M^{2}$ plots (the so-called Arrott plots) that are shown in Fig. 3. We recall that in an Arrott plot, where $H / M$ is plotted against $M^{2}$, the curvature is expected to change at a specific temperature, where the magnetic ordering transition takes place. In the present case, there clearly exist three characteristic temperature ranges at which the shape of Arrott plots changes sharply, as shown in Fig. 3 for representative temperatures. Like $H / M(T)$, the Arrott plots indicate a ferrimagnetic transition at $\sim 240 \mathrm{~K}$. A notable change in the curvature is seen at temperatures below $225 \mathrm{~K}$, which is associated with the onset of the glassy behavior. The rapid change in the curvature below $170 \mathrm{~K}$ is attributed to the magnetic transition which is associated with the structural transition. ${ }^{9}$ A further change in the curvature is noted in Fig. 3 for $T \leq 55 \mathrm{~K}$, where all spins become completely frozen. Furthermore, the increase in the coercivity $\left(H_{C}\right)$ with decreasing temperature below $225 \mathrm{~K}$ (see inset of Fig. 3) is consistent with the fact that the cluster size increases as the temperature is lowered.

Work at USF was supported by DOE BES Physical Behavior of Materials Program through Grant No. DE-FG0207ER46438. Research at ORNL was sponsored by the Division of Materials Sciences and Engineering, Office of Basic Energy Sciences, U.S. Department of Energy.

${ }^{1}$ S.-W. Cheong and M. Mostovoy, Nature Mater. 6, 13 (2007).

${ }^{2}$ N. Ikeda, H. Ohsumi, K. Ishii, T. Inami, K. Kakurai, Y. Murakami, K. Yoshii, S. Mori, Y. Horibe, and H. Kito, Nature (London) 436, 1136 (2005).

${ }^{3}$ Y. Zhang, H. X. Yang, Y. Q. Guo, C. Ma, H. F. Tian, J. L. Luo, and J. Q. Li, Phys. Rev. B 76, 184105 (2007)

${ }^{4}$ M. Angst, R. P. Hermann, A. D. Christianson, M. D. Lumsden, C. Lee, M.-H. Whangbo, J.-W. Kim, P. J. Ryan, S. E. Nagler, W. Tian, R. Jin, B. C. Sales, and D. Mandrus, Phys. Rev. Lett. 101, 227601 (2008).

${ }^{5}$ M. A. Subramanian, T. He, J. Chen, and N. S. Rogado, T. G. Calvarese, and A. W. Sleight, Adv. Mater. (Weinheim, Ger.) 18, 1737 (2006).

${ }^{6}$ H. J. Xiang and M.-H. Whangbo, Phys. Rev. Lett. 98, 246403 (2007).

${ }^{7}$ S. Funahashi, J. Akimitsu, K. Shiratori, N. Kimizuka, M. Tanaka, and H. Fujishita, J. Phys. Soc. Jpn. 53, 2688 (1984).

${ }^{8}$ J. Iida, M. Tanaka, Y. Nakagawa, S. Funahashi, N. Kimizuka, and S. Takekawa, J. Phys. Soc. Jpn. 62, 1723 (1993)

${ }^{9}$ A. D. Christianson, M. D. Lumsden, M. Angst, Z. Yamani, W. Tian, R. Jin, E. A. Payzant, S. E. Nagler, B. C. Sales, and D. Manddrus, Phys. Rev. Lett. 100, 107601 (2008).

${ }^{10}$ F. Wang, J. Kim, Y. J. Kim, and G. D. Gu, arXiv:0712.1975 (unpublished).

${ }^{11}$ Spin Glasses and Random Fields, Series on Directions in Condensed Matter Physics Vol. 12, edited by A. P. Young (World Scientific, Singapore, 1998).

${ }^{12}$ S. Mukherjee, R. Ranganathan, P. S. Anilkumar, and P. A. Joy, Phys. Rev. B 54, 9267 (1996).

${ }^{13}$ D. N. H. Nam, K. Jonason, P. Nordblad, N. V. Khiem, and N. X. Phuc, Phys. Rev. B 59, 4189 (1999).

${ }^{14}$ P. C. Hohenberg and B. I. Halperin, Rev. Mod. Phys. 49, 435 (1977).

${ }^{15}$ K. Mukherjee and A. Banerjee, Phys. Rev. B 77, 024430 (2008). 